\title{
Cell Imaging by Spontaneous and Amplified Raman Spectroscopies
}

\author{
Giulia Rusciano, ${ }^{1,2}$ Gianluigi Zito, ${ }^{3}$ Giuseppe Pesce, ${ }^{1}$ and Antonio Sasso ${ }^{1,2}$ \\ ${ }^{1}$ Department of Physics "E. Pancini”, University of Naples Federico II, Via Cintia, 80126 Naples, Italy \\ ${ }^{2}$ National Institute of Optics (INO), National Research Council (CNR), Via Campi Flegrei 34, 80078 Pozzuoli, Italy \\ ${ }^{3}$ Institute of Protein Biochemistry (IBP), National Research Council (CNR), Via Pietro Castellino 111, 80131 Napoli, Italy \\ Correspondence should be addressed to Giulia Rusciano; giulia.rusciano@unina.it
}

Received 28 September 2016; Accepted 27 December 2016; Published 27 February 2017

Academic Editor: Renata Diniz

Copyright ( 2017 Giulia Rusciano et al. This is an open access article distributed under the Creative Commons Attribution License, which permits unrestricted use, distribution, and reproduction in any medium, provided the original work is properly cited.

\begin{abstract}
Raman spectroscopy (RS) is a powerful, noninvasive optical technique able to detect vibrational modes of chemical bonds. The high chemical specificity due to its fingerprinting character and the minimal requests for sample preparation have rendered it nowadays very popular in the analysis of biosystems for diagnostic purposes. In this paper, we first discuss the main advantages of spontaneous RS by describing the study of a single protozoan (Acanthamoeba), which plays an important role in a severe ophthalmological disease (Acanthamoeba keratitis). Later on, we point out that the weak signals that originated from Raman scattering do not allow probing optically thin samples, such as cellular membrane. Experimental approaches able to overcome this drawback are based on the use of metallic nanostructures, which lead to a huge amplification of the Raman yields thanks to the excitation of localized surface plasmon resonances. Surface-enhanced Raman scattering (SERS) and tip-enhanced Raman scattering (TERS) are examples of such innovative techniques, in which metallic nanostructures are assembled on a flat surface or on the tip of a scanning probe microscope, respectively. Herein, we provide a couple of examples (red blood cells and bacterial spores) aimed at studying cell membranes with these techniques.
\end{abstract}

\section{Introduction}

Optical methods are ideally suited for the analysis of biological systems because of the gentle light-matter interaction that makes them particularly noninvasive. Fluorescencebased techniques have been extensively employed for optical imaging of biological systems due to its high sensitivity. Nevertheless, these techniques suffer significant limitations mainly related to (i) photobleaching and photoblinking of the fluorescent labels, (ii) perturbation of the molecules induced by the fluorophores themselves, and (iii) poor chemical selectivity. Raman spectroscopy (RS) is attracting considerable attention in life sciences [1-3], because it overcomes these limitations and provides additional advantages, related to the recently explored possibility of improving the spatial resolution up to the nanoscale. RS is not affected by fluorescence quenching mechanisms being based on inelastic scattering of light; it does not require any sample preparation (label-free technique), and, further more important, it assesses the chemical composition of the sample by exploiting the unique vibrational structure of molecules (chemical fingerprinting). In principle, Raman spectra provide information similar to infrared (IR) absorption spectra, but with the important advantage of avoiding the strong IR absorption of water, which usually is dominant in biological samples (water, instead, exhibits a low Raman activity). These advantages have led to a remarkable development of the various types of Raman-based spectroscopies and their diffusion in many fields of science (biomedicine [4-6], cultural heritage [7], food control [8], forensics analysis [9], etc.). In addition, the combination of Raman spectroscopy with confocal scanning microscopy has allowed a chemical imaging of tissues or even of single cells (Raman imaging) [6]. Moreover, changes in molecular composition of biosystems, detectable using multivariate statistical approaches, enable quantitative diagnosis of diseases with high level of sensitivity and specificity $[10,11]$.

However, also Raman spectroscopy has several drawbacks. The most severe limit of RS is the relatively low 
efficiency of the inelastic light-scattering process. As a matter of fact, RS cross section is typically $10^{-30}-10^{-25} \mathrm{~cm}^{2} \mathrm{sr}^{-1}$, that is, $8-10$ orders of magnitude weaker than the fluorescence/ absorption cross section. Thus, RS fails in detecting molecules at low concentration (below $0.1 \mathrm{mM}$ ). This drawback can be overcome by several Raman-based techniques, including resonant Raman spectroscopy (RRS) [12], coherent antiStokes Raman spectroscopy (CARS) [13], or stimulated Raman spectroscopy (SRS) [14], which have been developed to enhance the intensity and improve the spatial resolution with respect to the basic method.

A quite efficient way to enhance the weak signals in spontaneous RS is based on the lucky combination with plasmonics, which takes advantage of the coupling of light to metallic nanostructures. Such combination is at the basis of surface-enhanced Raman scattering (SERS). Historically, SERS birth dates back to 1974 when Fleischmann and coworkers [15] observed an anomalous, huge enhancement of the Raman signal from pyridine molecules adsorbed on a roughened silver electrode. A few years later, two independent papers $[16,17]$ provided the first physical interpretation based on localized surface plasmon resonances (LSPRs). High local electromagnetic field near the nanostructures provides very high enhancement of Raman scattering. The maximum SERS enhancement factor (EF) occurs at specific positions on the surface called hot spots. When molecules are adsorbed to or in close proximity (within a few $\mathrm{nm}$ ) of a hot spot, a huge Raman enhancement up to 12 orders of magnitude can occur, allowing single-molecule sensitivity $[18,19]$. The SERS EF depends on several parameters, such as the real and imaginary parts of the dielectric function of the metal nanostructure, the active substrate's geometry, the metal-molecule affinity, and the possible presence of electronic transition resonances of the analyte molecule at the excitation wavelength. Despite such complexity, SERS has found successful application in cutting-edge biochemical and medical research [20-22]. In particular, SERS technique has proved to be an effective platform for optical probing and imaging of live cells via SERS nanotags or in label-free experiments for surface cell sensing [23-29]. In this context, metal colloids have found wide application as SERS-active substrate, due to the particularly simple technology required for colloid preparation. Nevertheless, colloidal SERS substrates suffer from insufficient spatial reproducibility due to their local geometric heterogeneity that induces an uncontrollable position-dependent enhancement. Although great efforts have been dedicated in the last few decades to the synthesis and fabrication of high performance SERS substrates in terms of sensitivity and reproducibility, this topic is still open.

The unpredictable localization of the hot spots clearly undermines the potential subdiffraction character of SERS which, in fact, remains limited to the size of the focused laser spot. This limit can be overcome by combining the features of SERS with those of scanning probe microscopies, like Atomic Force Microscopy (AFM) and Scanning Tunnelling Microscopy (STM). This technique is called tip-enhanced Raman scattering (TERS) and is implemented by illuminating a metallic (or metaled) tip by a tightly focused laser beam.

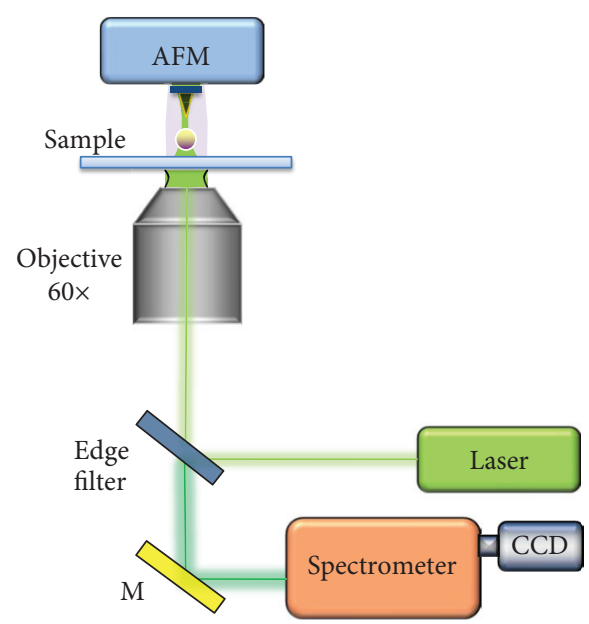

FiguRE 1: Schematic picture of our Raman set-up. Spontaneous RS or SERS measurements are made in backscattering configuration. In the upper part is mounted the AFM head for TERS measurements.

The metal tip acts then as a highly localized nanoantenna able to provide a strong amplification of the Raman signal via LSPR excited at the tip apex. In such a way, TERS analysis provides, simultaneously, the topographical (based on scanning microscopy data) and chemical (based on SERS effect at the tip apex) information of the investigated surface [30, 31]. TERS technique has, in very recent years, allowed a deeper insight into the surface properties of many systems at the nanoscale, including biosystems [32-36].

The scope of this paper is not to provide a description of the physical principles of the mentioned techniques (for that we refer to textbook and papers listed in the references). Rather, this paper reviews some specific cases studied aimed at providing Raman cell imaging at different levels of complexity and spatial resolution. The starting point is the study of a single protozoan based on spontaneous RS; we show how $\mathrm{RS}$, combined with principal component analysis (PCA), is able to distinguish different regions of the cell and their evolution when the cell is exposed to an external stress. Then, after a brief description of a new approach to fabricate high uniform nanostructured metallic substrates, we demonstrate that SERS provides information on the distribution of chemicals on the membrane of red blood cells (RBCs). Finally, we conclude the review by discussing the study of the bacterial spore surface using TERS and demonstrating the high spatial resolution and chemical selectivity of this technique.

\section{Material and Methods}

2.1. Raman Confocal Microscopy. All the experiments described herein have been performed by using an inverted Raman confocal microscope (WiTec, alpha 300). The system, schematically shown in Figure 1, is equipped with an excitation source at $532 \mathrm{~nm}$, provided by a frequency doubled Nd:YAG laser. Raman scattered light is collected with the same objective lens used to focus the radiation onto the sample and is guided to the spectrograph by a $25 \mu \mathrm{m}$ core optical fiber, which assures the system confocality. Spectra 
are acquired using a 600 and $1800 \mathrm{l} / \mathrm{mm}$ grating, according to the requested resolution. The system is combined with an Atomic Force Microscope for TERS measurements.

2.2. SERS Substrate Preparation. The procedure to obtain our SERS substrates is based on the self-assembling of block-copolymer (BCP) micelles of polystyrene-block-poly4-vinylpyridine (PS-b-P4VP, molecular weight 10400-b19200) loaded with silver nanoparticles (Ag-NPs) which is described in [37]. It is based on the procedure firstly described in [38], properly modified to increase the nanoislands' packing density (see Figure 2). Briefly, a concentrated Ag-BCP solution was processed with centrifugation, filtration, and slow spin coating over $24 \mathrm{~mm} \times 24 \mathrm{~mm}$ commercial coverslips. Polymers were removed from the thin film after UV exposure. In such way, we obtained a self-assembled isotropic nanostructure with characteristics of homogeneity typical of the so-called near hyperuniform disorder $[39,40]$. The resulting nanoisland pattern exhibited a hot spot density $\sim 10^{4} \mu \mathrm{m}^{-2}$. To test the spatial reproducibility and the enhancement factor of such plasmonic structure, we used a common dye (crystal violet) as probe molecule. We found a very high spatial reproducibility (standard deviation $<1 \%$ over $2500 \mu \mathrm{m}^{2}$ ) and a high enhancement factor (EF up to $\sim 10^{8}$ ) suitable for single-molecule detection.

\section{Cells Preparation and Handling}

3.1. Acanthamoeba. Acanthamoeba cysts (A.) were isolated from the cornea of contact lens user following a procedure described in [41]. Protozoa were suspended in Page's amoeba saline and centrifuged at $800 \mathrm{~g}$ for $5 \mathrm{~min}$; therefore, cells were isolated in 1.5\% nonnutrient agar enriched by Escherichia coli K12. Sample preparation was carried out by dipping PBS solution containing cells into a glass chamber, consisting of two sandwiched coverslips glued by two parafilm strips also acting as spacers. Raman analysis was performed on cells spontaneously adhered onto the bottom glass coverslip.

3.2. Red Blood Cells. RBCs directly extracted by healthy donors were stored in an isotonic solution for $30 \mathrm{~min}$ prior to the experiments with no further treatment. Therefore, cells were infiltrated into a cell chamber, similar to that previously described for $A$. cysts. For SERS measurements, the chamber bottom coverslip was replaced by a SERS substrate, prepared according to the procedure previously described. For both spontaneous Raman and SERS imaging, RBCs were left to adhere by physisorption to the chamber bottom for $\sim 1$ hour before analysis. Importantly, we did not observe hemolytic activity of our immobilized SERS nanostructure on the time scale of the measurements.

3.3. Bacterial Spores. TERS analysis was performed on Bacillus subtilis (B. subtilis) spores. The sample was prepared by pipetting $50 \mu \mathrm{L}$ of a water suspension of mature PY79 wild-type spores at a concentration of $10^{5}$ spores $/ \mathrm{mL}$ on a clean microscope glass coverslip and left to dry at ambient conditions.

\section{Results and Discussion}

4.1. Spontaneous Raman Imaging of a Single Protozoan. Acanthamoeba (A.) keratitis is a severe eye infection, often affecting contact lens wearers, which can lead to permanent visual impairment or even blindness. Diagnosing $A$. keratitis is a clinical challenge. Nowadays, it is performed by the association of a microscopic examination of the corneal scraping, culture of parasites, and molecular biology assays based on DNA amplification such as Polymerase Chain Reaction (PCR). Clinical prescriptions for $A$. keratitis often involve the use of Polyhexamethylene Biguanide (PHMB), an antiseptic toxic for the epithelial cells of the cornea. This renders a fundamental timely suspension of the drug therapy when protozoa are eradicated. For this reason, innovative tools to distinguish between living and dead $A$. are strongly required. We have tested this capability for Raman imaging.

To get a Raman image of a single $A$., spectra were acquired in a raster scan around the cell. Measurements were performed keeping $A$. in a simple PBS solution or in presence of PHMB (concentration 1:2 in PBS). The complexity of the obtained spectra, exemplified in Figure 3(a), rendered necessary to analyze signals by PCA [42]. At this purpose, the acquired spectra were background-corrected by removing a fourth-order polynomial and eliminating spurious signals deriving from cosmic ray contributions. Therefore, they were processed by using a homemade MATLAB routine. Figure 3 shows the PC1 score maps reconstructed for the same $A$., before (Figure 3(b)) and after 6 hours of exposure to PHMB (Figure 3(c)). PC1 loading presents the characteristic DNA bands at 728,785 , and $1570 \mathrm{~cm}^{-1}$ (data not shown; see [43] for details) indicating therefore that the PC1 score map is related to the nucleic acid distribution. Interestingly, while before treatment with PHMB the high score values are localized in a well-defined region within the cell, after exposure to $\mathrm{PHMB}, \mathrm{DNA}$ is distributed in a much wider region, therefore suggesting the rupture of nuclear membrane and the successive diffusion of nuclear material throughout the cell. PC2 loading features, instead, can be associated with the cell membrane. In particular, spatial localization of PC2 scores gives evidence of membrane rupture induced by exposition to $\mathrm{PHMB}$.

4.2. SERS Imaging of Red Blood Cell Membrane. Label-free chemical imaging of live cell membranes can shed light on the molecular basis of cell membrane functionalities and their alterations under pathological conditions. For instance, it is well known that, in the presence of a malignant cell transformation, the membrane biochemical composition is strongly modified. However, spontaneous Raman spectroscopy is unable to reach this goal. As a matter of fact, due to the limit imposed by the diffraction of light, scattered Raman photons originate from a volume whose extension, in the Raman probe propagation direction $z$, is typically a couple of microns. Hence, the confocal detection region includes a relatively thick cytosolic region, which often renders negligible the contribution of the nanometric membrane layer. Therefore, techniques capable of introducing not only an axial spatial selectivity but also an adequate sensitivity to 


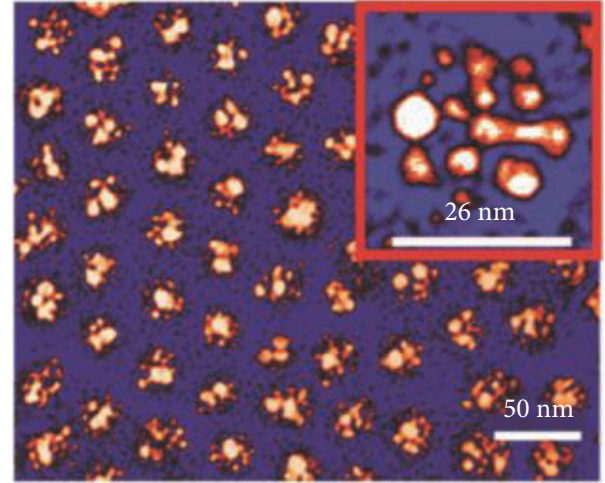

(a)

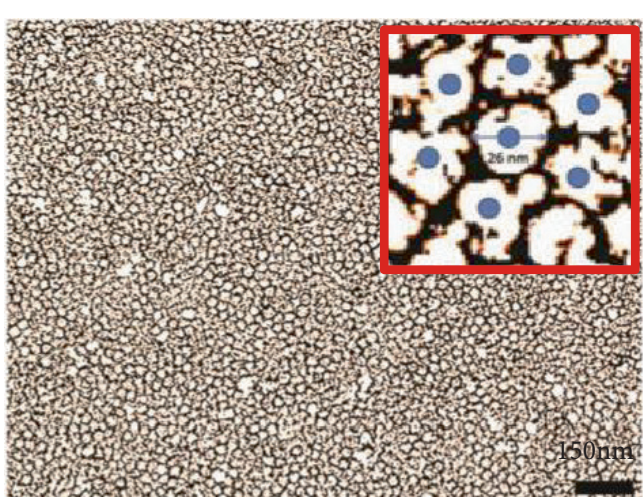

(b)

FIgURE 2: Characterization of the SERS substrate. (a) TEM micrograph of hexagonal nanostructure obtained following the procedure described in [25]. The inset shows, in detail, a single cluster of Ag-NPs. (b) TEM micrograph of the pattern obtained by applying our implemented protocol. The filling fraction is highly increased reducing nanoisland gaps to 2-3 nm, as shown in the inset. Bar scales are $50 \mathrm{~nm}$ in (a) and $150 \mathrm{~nm}$ in (b). Bar scale in the inset in (a) is $26 \mathrm{~nm}$. (Adapted from [37] with permission from the Royal Society of Chemistry.)

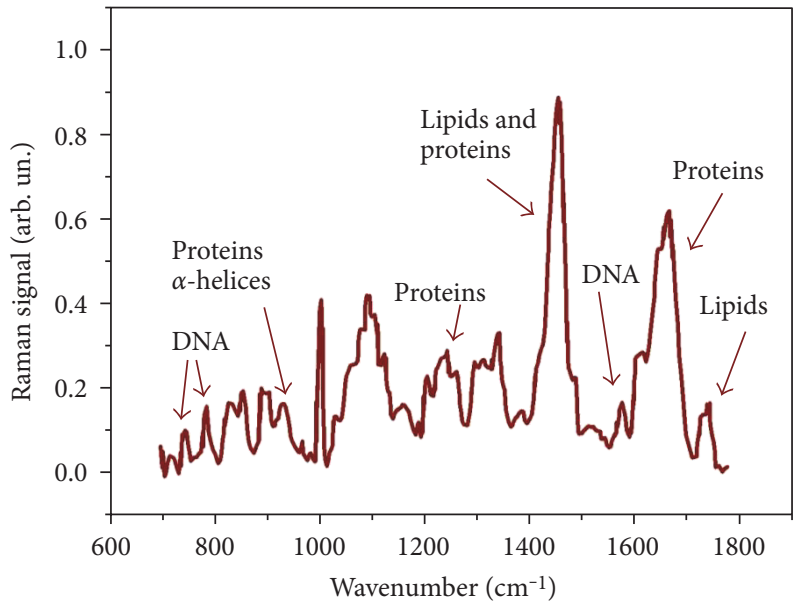

(a)

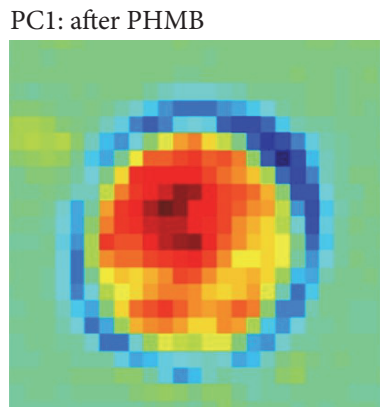

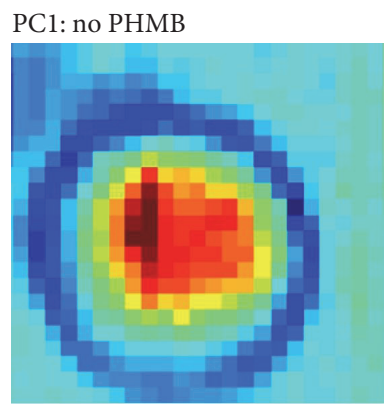

Bright field

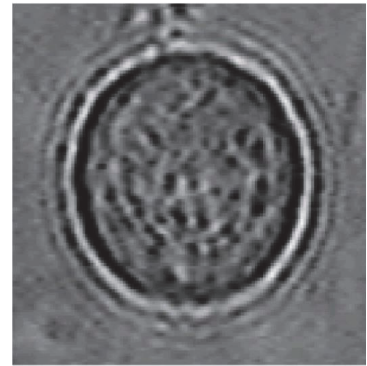

(b)

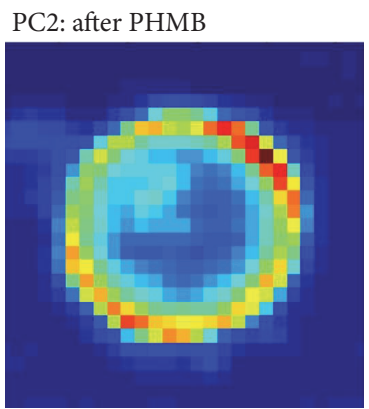

(c)

Figure 3: (a) A typical Raman spectrum from a live Acanthamoeba. (b) Score maps of PC1 component for an A., before exposure to PHMB (left) compared with its bright field image (right). (c) Score map of PC2 (left) and PC3 (right) components after 6 hours of A. exposure to $\mathrm{PHMB}$.

reveal a reduced number of molecules are strongly required. SERS fulfills both requirements, thanks to the giant enhancement of the signal corresponding to molecules in close contact with the plasmonic substrate. In order to demonstrate the effectiveness of SERS for membrane analysis, we applied this technique to a challenging case of study: the RBC. As a matter of fact, a RBC consists of a closed lipid bilayer membrane (embedding proteins) acting as a bag for hemoglobin 

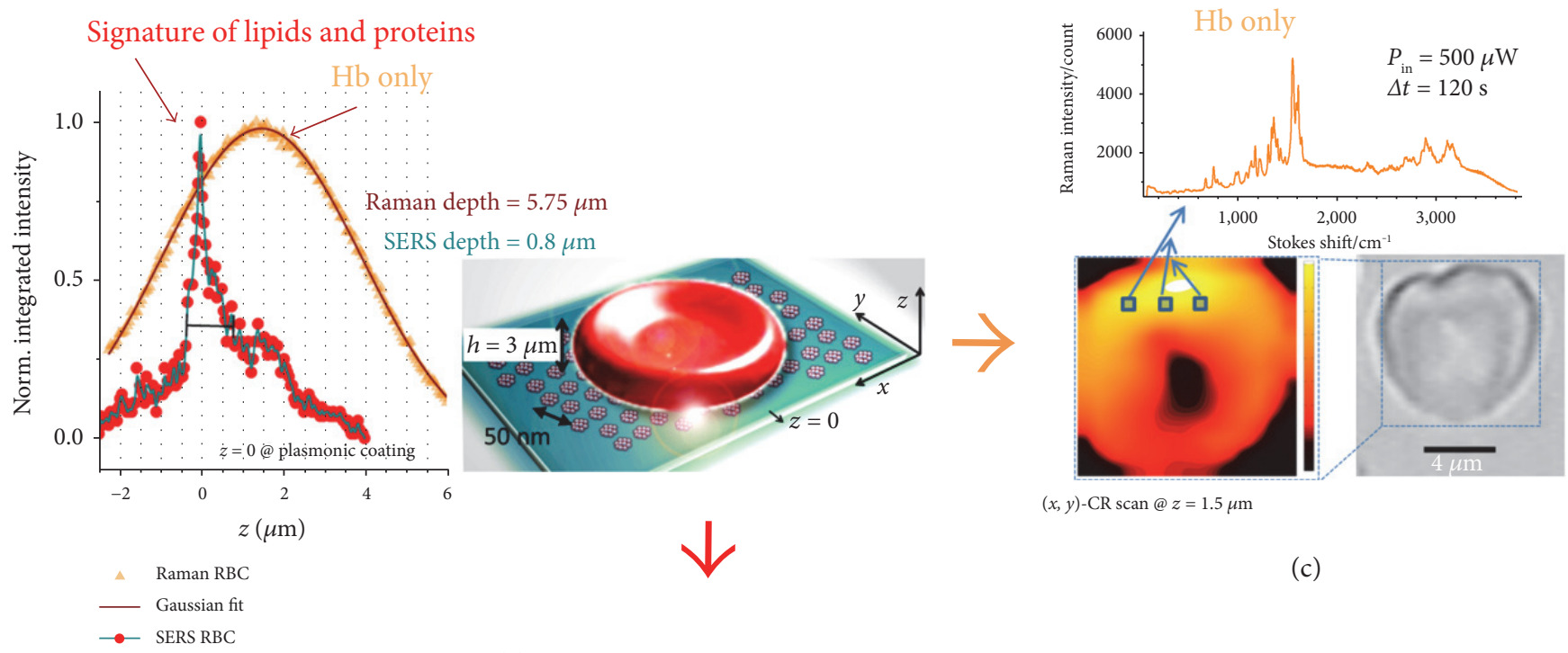

$(x, y)$-CR scan @ $z=1.5 \mu \mathrm{m}$

(a)

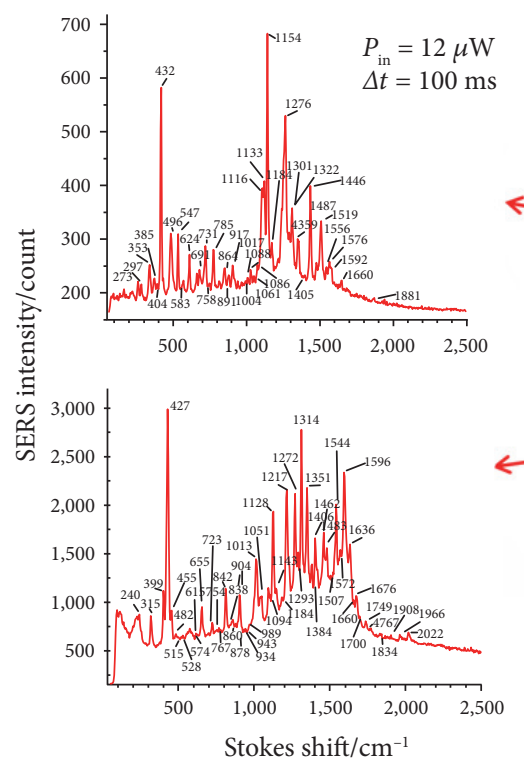

Signature of lipids and proteins (not masked of $\mathrm{Hb}$ ) $(x, y)$-SERS scan @ $z=0$

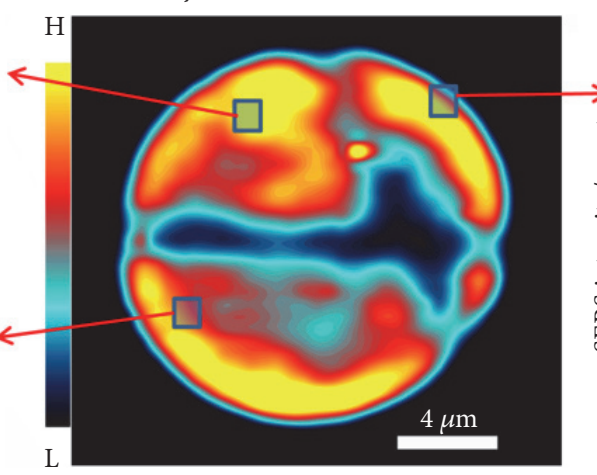
with spatial resulotion

(c)

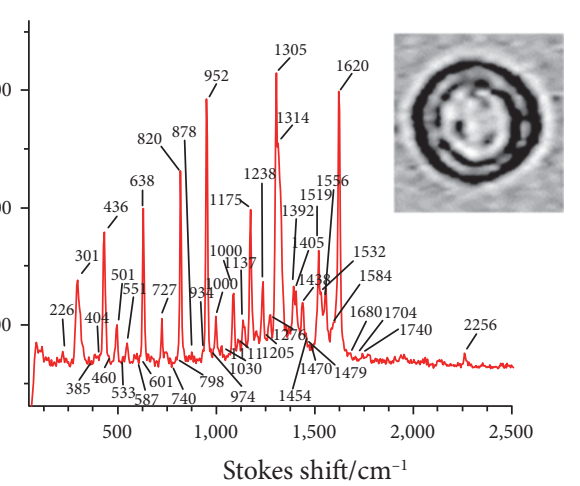

(b)

FIGURE 4: Schematic representation of the experiment with red blood cell on a SERS substrate: the SERS signal is peaked at the SERS substrate $z=0$ in (a) (red points) and is associated with SERS spectra corresponding to lipids and proteins not masked by Hb as shown in (b). In particular, this is achieved with spatially resolved signals revealing sensitivity to the local membrane environment with capability of reconstructing the membrane SERS map of the RBC, in close correlation with the actual morphology of the cell scanned (see optical image in the inset in (b)). In a twin experiment with a normal coverslip as substrate (not shown), the Raman signal is ascribable only to $\mathrm{Hb}$ : the spontaneous Raman intensity (integrated between 1400 and $1600 \mathrm{~cm}^{-1}$ ) versus the axial distance $z$ denotes a broad bell-shaped curve (orange points) to be contrasted to the sharp and localized curve obtained on SERS substrate (red points in (a)). In this case, Raman imaging reveals only the spectral signature of $\mathrm{Hb}$ in all the RBC volume with no contribution from membrane, as represented in (c).

(Hb). This latter protein presents spectral resonance for excitation in the visible region, which clearly introduces an additional level of complexity for the spectral detection of scattering from the membrane.

In order to unambiguously assign the observed signals to SERS scattering from the RBC plasma membrane in contact with the Ag nanotextured substrate, we proceeded with two twin experiments. In the first experiment, RBCs extracted following the protocol described in the previous paragraph were placed on a chamber closed at the bottom with a simple glass coverslip. In the second experiment, the glass coverslip was replaced by a SERS substrate (Figure 4(a)). In both cases, RBCs were allowed to spontaneously adhere to the chamber bottom. Therefore, we repeatedly acquired Raman spectra at different heights by scanning the sample along the $z$-axial coordinate. Marked 
differences were found. In particular, for the spontaneous Raman case, spectra were mainly ascribable to the bulk hemoglobin contained inside the cell, presenting typical spectral bands of the heme group [44]. At the same time, the signal intensity (integrated over the $600-1800 \mathrm{~cm}^{-1}$ region) versus the $z$ coordinate was characterized by a broad bell shape $(\sim 5.8 \mu \mathrm{m}$ FWHM), consistent with RBC thickness convoluted with the $z$-axis resolution. On the contrary, in the second case, no $\mathrm{Hb}$ contribution was observed and the signal intensity versus $z$ (distance from the substrate) presented a much sharper curve, with a maximum at $z=0$ and an effective depth $(\sim 0.8 \mu \mathrm{m})$ consistent with the effective thickness of the confocal scattering volume.

Since membrane thickness is limited to $\sim 5 \mathrm{~nm}$, and whereas the signal decays rapidly with distance from the surface substrate, we believe that we are actually revealing the only contribution from the outermost part of the membrane. Typical SERS spectra are reported in Figure 4(b). The spectra were clearly dominated by spectral features due to lipids, amino acids, and carbohydrates with no typical markers of membrane-bound $\mathrm{Hb}$.

A prerequisite for a faithful SERS imaging is the availability of plasmonic architectures with a spatially invariant SERS enhancement factor. This requirement has opened new research area in nanoplasmonic, aimed at producing nanostructured surface showing high efficiency and reproducibility. We faced this problem by taking advantage of the use of the so-called block copolymer (BCP), polymers made up of blocks of different monomeric components. Importantly, when spun on a glass coverslip, BCP tends to self-assemble at nanoscale, giving rise to nanotextured coverslip coating, whose features depend on their concentration and molecular weight. In our case, starting from BCP micelles loaded with silver nanoparticles (Ag-NPs), we obtained a self-assembled isotropic nanostructure with characteristics of homogeneity typical of the so-called near-hyperuniform disorder. The resulting highly dense, homogeneous, and isotropic random pattern consists of clusters of silver nanoparticles with limited size dispersion.

A raster scan SERS imaging was performed by acquiring spectra on a square grid, with a step of $200 \mathrm{~nm}$, close to the spatial resolution in the $x-y$ plane. The result is shown in Figure 4(b) and is indicated as SERS map. We observed variations of the SERS spectrum depending on the membrane position, denoting a sensitivity to the local molecular environment explored in the laser-scattering area. The representative hyperspectral map, integrated in the range $1100-1700 \mathrm{~cm}^{-1}$, reveals a strong correlation with the optical image of the same RBC (see Figure 4(b), optical image on the right). Clearly, such faithful SERS imaging was actually possible because of the excellent uniformity of the enhancement factor of our nanostructure.

4.3. TERS Imaging of Bacterial Spore Membrane. For most of the applications of Raman analysis to biosystems, the intrinsic diffraction-limited spatial resolution of this technique fits the experiment requirements. However, for some specific cases, mainly related to the analysis of single nanometric targets (DNA filaments, fibrils, etc.), it is required to beat the diffraction limit. TERS analysis is the answer for this demand [45].

In this section, we show an application of this technique to a quite interesting biosystem: the Bacillus subtilis (B. subtilis) spore. These spores have recently been demonstrated to hold potential for many biotechnological applications as the drug delivery [46] or the development of new sources for energy production [47]. We have applied TERS analysis to the spore's outmost layer (the coat), focusing our attention on particular surface-nanosized structures, the so-called spore ridges [48], readily distinguishable in topographic AFM images of spores (see Figure 5(a)). The function of these structures is still not fully understood, although it is surely related to the volume changes occurring during spore core dehydration-rehydration cycles.

Figure 5(b) shows a typical TERS spectrum observed in this investigation. It was obtained by a laser power impinging on the sample of $50 \mu \mathrm{W}$ and an integration time and $2 \mathrm{~s}$. The complexity of this spectrum reflects the intricate arrangements of proteins, lipid, and carbohydrates on the spore surface. Importantly, the acquired spectra exhibited significant point-to-point variations, reflecting the different nanoenvironments explored by the tip. In this condition, obtaining useful information from TERS data is surely a challenge. Even more difficult is to highlight the correlations of TERS data with the observables provided by other nanoscopic techniques. In particular, it is reasonable to expect a strong correlation between AFM phase maps and TERS maps, being both surface representations sensitive to the surface chemical signatures. However, TERS maps reporting the intensity of selected spectral features do not provide useful information and seem to be completely uncorrelated by AFM phase maps. For instance, Figure 5(c) shows a $120 \mathrm{~nm} \times 120 \mathrm{~nm}$ phase map, obtained with a scan step of $20 \mathrm{~nm}$. The brighter pixels (corresponding to a positive phase lag) correspond to points on the ridge. Figure 5(d), instead, reports TERS maps obtained by reporting the intensity of assigned spectral TERS bands. Evidently, no apparent correlation can be revealed between these maps and the phase map. However, information-rich TERS maps can be instead obtained by taking advantage of PCA. To test the effectiveness of this statistical tool for unravelling the information provided by TERS spectra of spore surface, we have acquired spectra in a spore-surface zone across a surface ridge clearly visible in the spore AFM phase map. However, a completely different outcome is obtained when TERS data are analyzed by PCA. In particular, as evident in Figure 5(e), both PC2 and PC3 score maps exhibit a clear correlation with the phase map ( $\mathrm{PCl}$ components only take into account a residual background, so it was not taken into consideration). Therefore, this study demonstrates that by taking advantage of advanced statistical tools like the principal component analysis, TERS maps can be correlated with AFM phase maps, thus allowing one to pick up some spectroscopic-derived outcomes nonintuitively identifiable. This might be very useful for drawing solid conclusions from the TERS analysis of complex organisms and, therefore, also support the diffusion of this technique in many fields of science, including biology and biomedicine. 


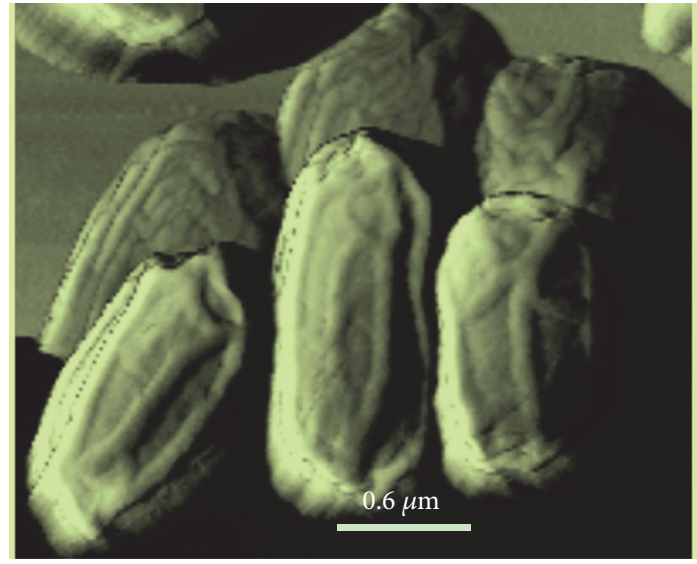

(a)

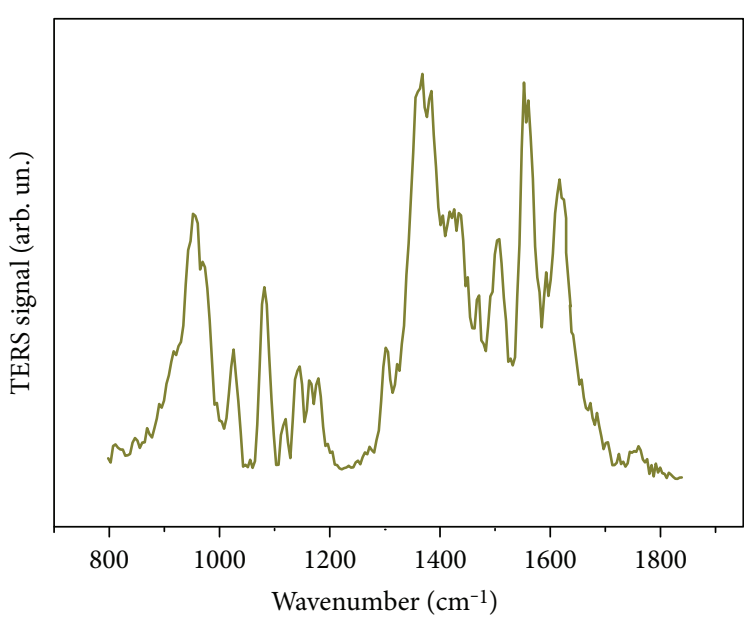

(b)

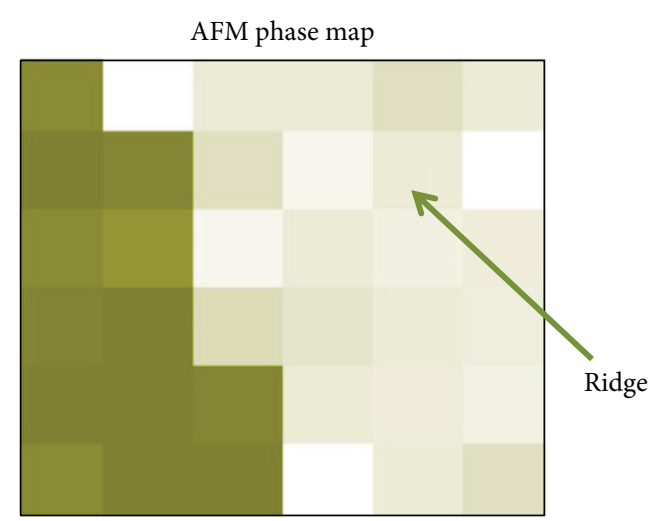

(c)

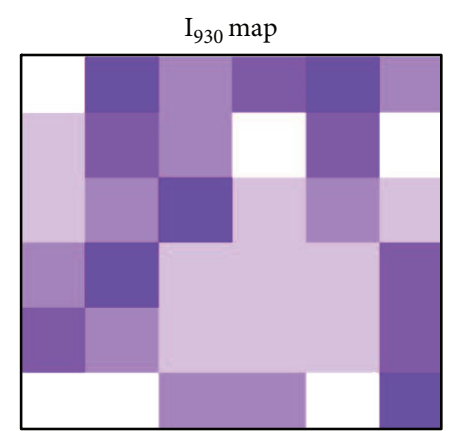

(d)

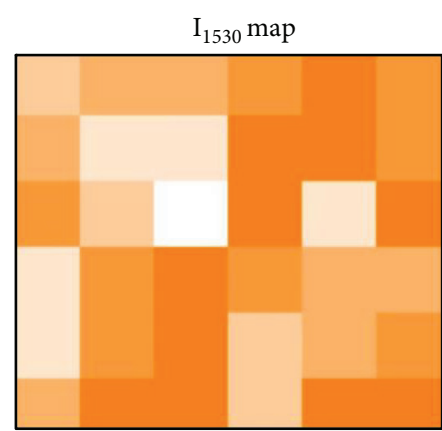

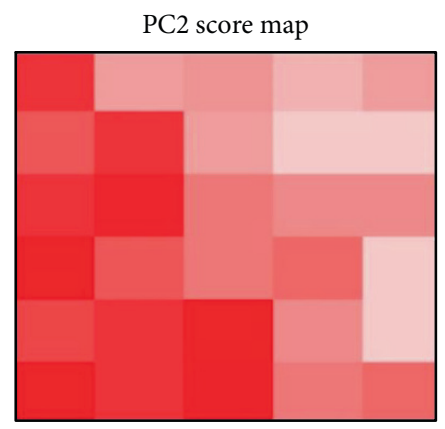

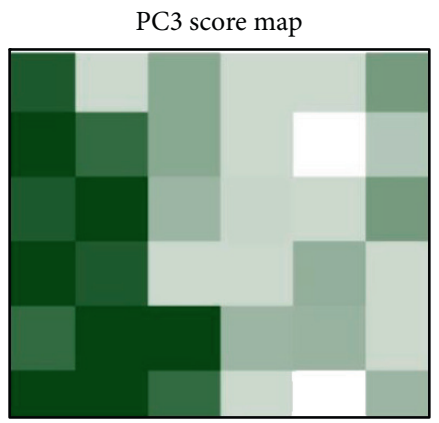

(e)

FIGURE 5: (a) AFM topographical image of fully dehydrated B. subtilis. Spore surface exhibits characteristic ridges resulting from volume contraction due to the core dehydration. (b) A typical TERS spectrum from the coat of a B. subtilis spore. (c) AFM phase map of a whole spore acquired in tapping mode. Bright (dark) zones correspond to regions of higher (lower) stiffness. (d) TERS maps obtained by reporting the intensity of selected spectral features, indicated in the labels. (e) Second and third PC score maps of TERS data.

\section{Conclusions}

Single cell Raman spectroscopy, thanks to its noninvasive, label-free, and fingerprint character, provides rich and intrinsic information of the cell (e.g., nucleic acids, protein, carbohydrates, and lipids), reflecting cellular, physiological, and pathological states. This study demonstrates the possibility of applying Raman microspectroscopy for analyzing in vitro single Acanthamoeba cells, in order to monitor, in real time, the effect of PHMB, a strong disinfectant and antiseptic also employed to treat $A$. keratitis. A key drawback of Raman spectroscopy is related to the low inelastic scattering cross section, so that spontaneous Raman signals are naturally weak. This review discusses recent research progress in significantly enhancing and improving the signal of spontaneous Raman spectroscopy, including surface-enhanced Raman scattering and tip-enhanced Raman scattering. In particular, the results obtained for two interesting case studies are reported. Globally, our results underline the great potential of Raman and plasmon- 
enhanced Raman techniques for the analysis of biosystems, even for diagnostic purposes.

\section{Competing Interests}

The authors declare that they have no competing interests.

\section{Acknowledgments}

This research was supported in part by the project FIRB 2012-RBFR12WAPY of the Italian Ministry for Education, University and Research (MIUR).

\section{References}

[1] F. Siebert and P. Hildebrandt, Instrumentation, in Vibrational Spectroscopy in Life Science, Wiley-VCH Verlag GmbH \& Co, KGaA, Weinheim, Germany, 2007.

[2] R. Smith, K. L. Wright, and L. Ashton, "Raman spectroscopy: an evolving technique for live cell studies," Analyst, vol. 141, no. 12, pp. 3590-3600, 2016.

[3] G. Rusciano, A. C. De Luca, A. Sasso, and G. Pesce, "Phasesensitive detection in Raman tweezers," Applied Physics Letters, vol. 89, no. 26, Article ID 261116, 2006.

[4] M. Ghomi, Ed., Applications of Raman Spectroscopy to Biology: from Basic Studies to Disease Diagnosis, IOS Press, 2012.

[5] S. Managò, C. Valente, P. Mirabelli et al., "A reliable Ramanspectroscopy-based approach for diagnosis, classification and follow-up of B-cell acute lymphoblastic leukemia," Scientific Reports, vol. 6, article 24821, 2016.

[6] A. C. De Luca, K. Dholakia, and M. Mazilu, "Modulated Raman spectroscopy for enhanced cancer diagnosis at the cellular level," Sensors, vol. 15, no. 6, pp. 13680-13704, 2015.

[7] F. Casadio, C. Daher, and L. Bellot-Gurlet, "Raman spectroscopy of cultural heritage materials: overview of applications and new frontiers in instrumentation, sampling modalities, and data processing," Topics in Current Chemistry, vol. 374, no. 5 , p. $62,2016$.

[8] Y. S. Li and J. S. Church, "Raman spectroscopy in the analysis of food and pharmaceutical nanomaterials," Journal of Food and Drug Analysis, vol. 22, no. 1, pp. 29-48, 2014.

[9] J. M. Chalmers, H. G. M. Edwards, and M. D. Hargreaves, Eds., Introduction and Scope, in Infrared and Raman Spectroscopy in Forensic Science (eds J. M. Chalmers, H. G. M. Edwards and M. D. Hargreaves), John Wiley \& Sons, Ltd, Chichester, UK, 2012.

[10] A. Zoubir, Ed., Raman Imaging: Techniques and Applications, vol. 168, Springer-Verlag, Berlin Heidelberg, 2012.

[11] I. Barman, N. C. Dingari, G. P. Singh, R. Kumar, S. Lang, and G. Nabi, "Selective sampling using confocal Raman spectroscopy provides enhanced specificity for urinary bladder cancer diagnosis," Analytical and Bioanalytical Chemistry, vol. 404, no. 10, pp. 3091-3099, 2012.

[12] A. C. De Luca, G. Rusciano, R. Ciancia et al., "Spectroscopical and mechanical characterization of normal and thalassemic red blood cells by Raman tweezers," Optics Express, vol. 16, no. 11, pp. 7943-7957, 2008.

[13] A. Zumbusch, G. R. Holtom, and X. S. Xie, "Threedimensional vibrational imaging by coherent anti-Stokes Raman scattering," Physical Review Letters, vol. 82, no. 20, pp. 4142-4145, 1999.
[14] F. Lu, D. Calligaris, O. Olubiyi et al., "Label-free neurosurgical pathology with stimulated Raman imaging," Cancer Research, vol. 76, no. 12, pp. 3451-3462, 2016.

[15] M. Fleischmann, P. J. Hendra, and A. J. McQuillan, "Raman spectra of pyridine adsorbed at a silver electrode," Chemical Physics Letters, vol. 26, no. 2, pp. 163-166, 1974.

[16] D. L. Jeanmaire and R. P. Van Duyne, "Surface Raman spectroelectrochemistry, part 1: heterocyclic, aromatic, and aliphatic amines adsorbed on the anodized silver electrode," Journal of Electroanalytical Chemistry, vol. 84, no. 1, p. 120, 1977.

[17] M. G. Albrecht and J. A. Creighton, "Anomalously intense Raman spectra of pyridine at a silver electrode," Journal of American Chemical Society, vol. 99, no. 15, pp. 52155217, 1977.

[18] K. Kneipp, Y. Wang, H. Kneipp et al., "Single molecule detection using surface-enhanced Raman scattering (SERS)," Physical Review Letters, vol. 78, no. 9, pp. 1667-1670, 1997.

[19] E. C. Le Ru and P. G. Etchegoin, "Single-molecule surfaceenhanced Raman spectroscopy," Annual Review of Physical Chemistry, vol. 63, pp. 65-87, 2012.

[20] S. Schlücker, "Surface-enhanced Raman spectroscopy: concepts and chemical applications," Angewandte Chemie, International Edition, vol. 53, no. 19, pp. 4756-4795, 2014.

[21] W. Xie and S. Schlücker, "Rationally designed multifunctional plasmonic nanostructures for surface-enhanced Raman spectroscopy: a review," Reports on Progress in Physics, vol. 77, no. 11, Article ID 116502, 2014.

[22] G. Rusciano, A. De Luca, G. Pesce et al., "Label-free probing of G-quadruplex formation by surface-enhanced Raman scattering," Analytical Chemistry, vol. 83, no. 17, pp. 68496855, 2011.

[23] J. H. Granger, N. E. Schlotter, A. C. Crawford, and M. D. Porter, "Prospects for point-of-care pathogen diagnostics using surface-enhanced Raman scattering (SERS)," Chemical Society Reviews, vol. 45, no. 14, pp. 3865-3882, 2016.

[24] Z. H. Kim, "Single-molecule surface-enhanced Raman scattering: current status and future perspective," Frontiers of Physics, vol. 9, no. 1, pp. 25-30, 2014.

[25] L. Yang, P. Li, and J. Liu, "Progress in multifunctional surfaceenhanced Raman scattering substrate for detection," RSC Advances, vol. 4, no. 91, pp. 49635-49646, 2014.

[26] S. Ding, J. Yi, J. Li et al., "Nanostructure-based plasmonenhanced Raman spectroscopy for surface analysis of materials," Nature Reviews Materials, vol. 1, 16021 pages, 2016.

[27] A. Bonifacio, S. Cervo, and V. Sergo, "Label-free surfaceenhanced Raman spectroscopy of biofluids: fundamental aspects and diagnostic applications," Analytical and Bioanalytical Chemistry, vol. 407, no. 27, pp. 8265-8277, 2015.

[28] G. Rusciano, A. C. De Luca, G. Pesce, and A. Sasso, "On the interaction of nano-sized organic carbon particles with model lipid membranes," Carbon, vol. 47, no. 13, pp. 29502957, 2009.

[29] C. Muehlethaler, M. Leona, and J. R. Lombardi, "Review of surface enhanced Raman scattering applications in forensic science," Analytical Chemistry, vol. 88, no. 1, pp. 152-169, 2016.

[30] B. S. Yeo, J. Stadler, T. Schmid, R. Zenobi, and W. H. Zhang, "Tip-enhanced Raman spectroscopy: its status, challenges and future directions," Chemical Physics Letters, vol. 472, no. 1, pp. 1-13, 2009. 
[31] B. S. Yeo, S. Maedler, T. Schmid, W. H. Zhang, and R. Zenobi, "Tip-enhanced Raman spectroscopy can see more: the case of cytochrome c," Journal of Physical Chemistry C, vol. 112, no. 13, pp. 4867-4873, 2008.

[32] E. A. Pozzi, M. D. Sonntag, N. Jiang, J. M. Klingsporn, M. C. Hersam, and R. P. Van Duyne, "Tip-enhanced Raman imaging: an emergent tool for probing biology at the nanoscale," ACS Nano, vol. 7, no. 2, pp. 885-888, 2013.

[33] R. Treffer, R. Bohme, T. Deckert-Gaudig et al., "Advances in TERS for biochemical applications," Biochemical Society Transactions, vol. 40, no. 4, pp. 609-614, 2012.

[34] T. Schmid, L. Opilik, C. Blum, and R. Zenobi, "Nanoscale chemical imaging using tip-enhanced Raman spectroscopy: a critical review," Angewandte Chemie, International Edition, vol. 52, no. 23, pp. 5940-5954, 2013.

[35] G. Sharma, T. Deckert-Gaudig, and V. Deckert, "Tipenhanced Raman scattering-targeting structure-specific surface characterization for biomedical samples," Advanced Drug Delivery Reviews, vol. 89, pp. 42-56, 2015.

[36] Z. D. Schultz, J. Marr, and H. Wang, "Tip enhanced Raman scattering: plasmonic enhancements for nanoscale chemical analysis," Nanophotonics, vol. 3, no. 1-2, pp. 91-104, 2014.

[37] G. Zito, G. Rusciano, G. Pesce, A. Dochshanov, and A. Sasso, "Surface-enhanced Raman imaging of cell membrane by a highly homogeneous and isotropic silver nanostructure," Nanoscale, vol. 7, no. 18, pp. 8593-8606, 2015.

[38] W. J. Cho, Y. Kim, and J. K. Kim, "Ultrahigh-density array of silver nanoclusters for SERS substrate with high sensitivity and excellent reproducibility," ACS Nano, vol. 6, no. 1, pp. 249-255, 2012.

[39] S. Torquato and F. Stillinger, "Local density fluctuations, hyperuniformity, and order metrics," Physical Review E, vol. 68, no. 4, Article ID 041113, 2003.

[40] C. De Rosa, F. Auriemma, C. Diletto et al., "Toward hyperuniform disordered plasmonic nanostructures for reproducible surface-enhanced Raman spectroscopy," Physical Chemistry Chemical Physics, vol. 17, no. 12, pp. 8061-8069, 2015.

[41] G. Rusciano, P. Capriglione, G. Pesce et al., "Raman microspectroscopy analysis in the treatment of Acanthamoeba keratitis," PloS One, vol. 8, no. 8, pp. e72127-e72135, 2013.

[42] G. Rusciano, "Experimental analysis of Hb oxy-deoxy transition in single optically stretched red blood cells," Physica Medica, vol. 26, no. 4, pp. 233-239, 2010.

[43] G. Rusciano, P. Capriglione, G. Pesce et al., "Raman-spectroscopy-based biosensing for applications in ophthalmology," SPIE Optical Sensors Book Series: Proceedings of the SPIE, vol. 8774, Article ID 87740A, 2013

[44] B. R. Wood and D. McNaughton, "Raman excitation wavelength investigation of single red blood cells in vivo," Journal of Raman Specroscopy, vol. 33, no. 7, pp. 517523, 2002.

[45] G. Zito, G. Rusciano, A. Vecchione et al., "Nanometal skin of plasmonic heterostructures for highly efficient near-field scattering probes," Scientific Reports, vol. 6, p. 31113, 2016.

[46] E. Ricca and S. M. Cutting, "Emerging applications of bacterial spores in nanobiotechnology," Journal of Nanobiotechnology, vol. 1 , no. 1 , p. 6, 2003.
[47] X. Chen, L. Mahadevan, A. Driks, and O. Sahin, "Bacillus spores as building blocks for stimuli responsive materials and nanogenerators," Nature Nanotechnology, vol. 9, no. 2, pp. 137-141, 2014.

[48] G. Rusciano, G. Zito, R. Isticato et al., "Nanoscale chemical imaging of Bacillus subtilis spores by combining tipenhanced Raman scattering and advanced statistical tools," ACS Nano, vol. 8, no. 12, pp. 12300-12309, 2014. 

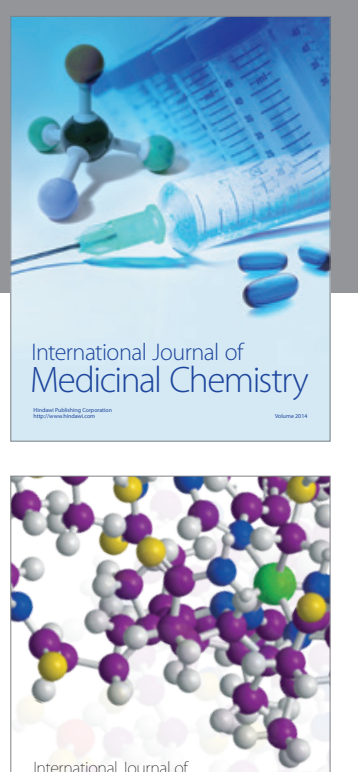

Carbohydrate Chemistry

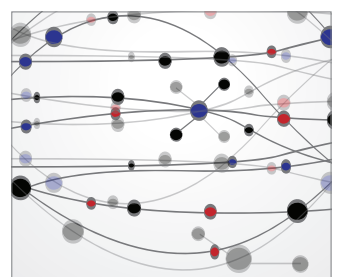

The Scientific World Journal
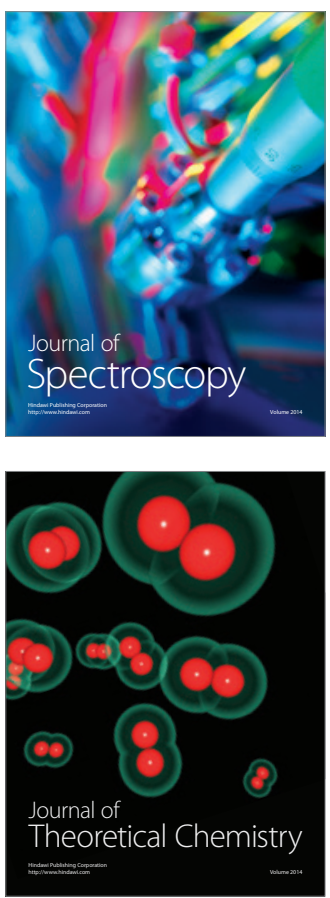
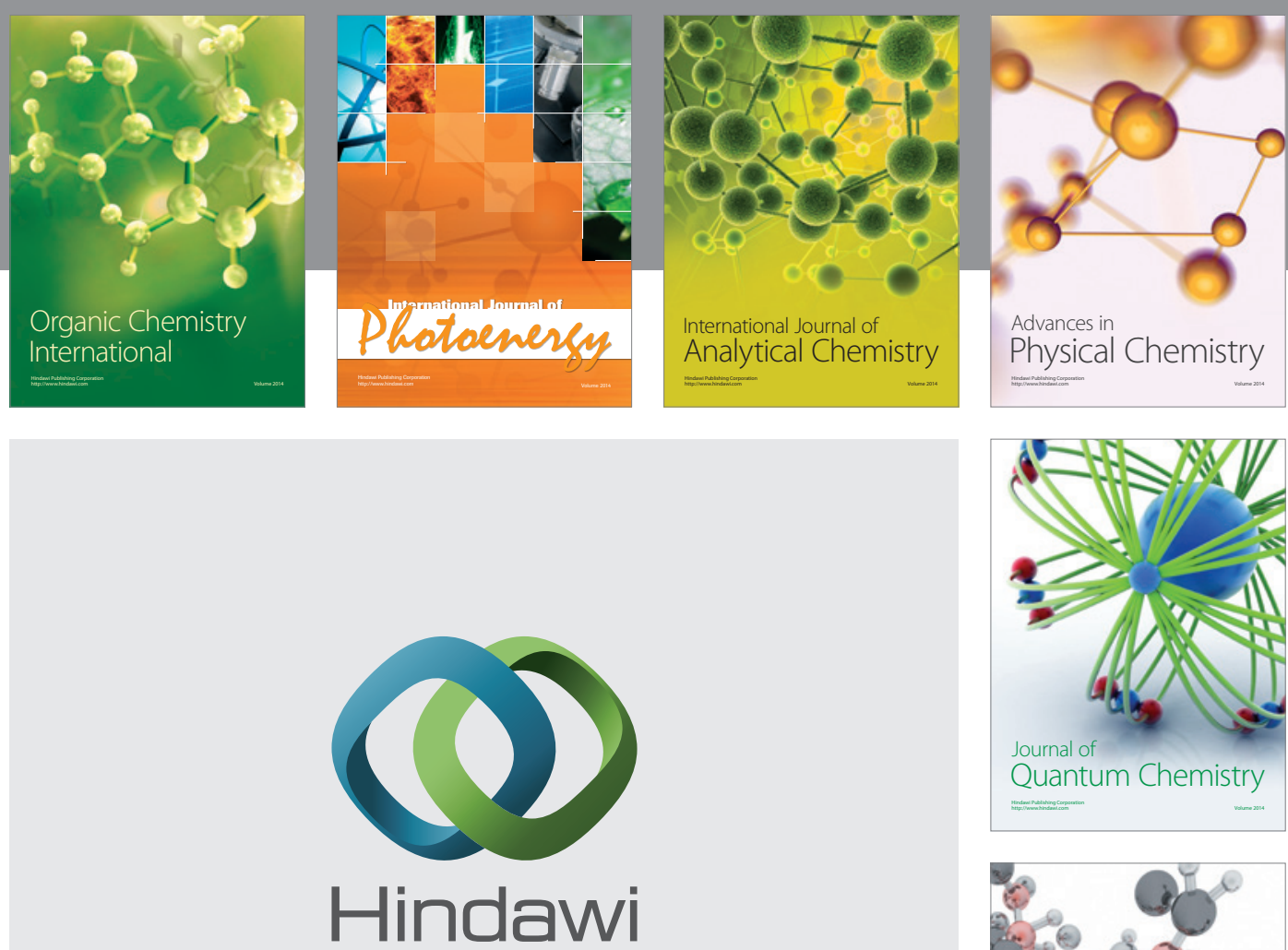

Submit your manuscripts at

https://www.hindawi.com

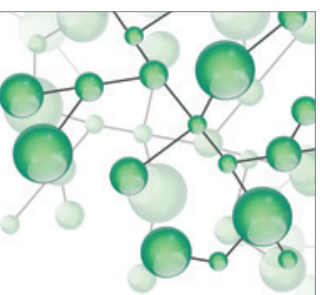

International Journal of

Inorganic Chemistry
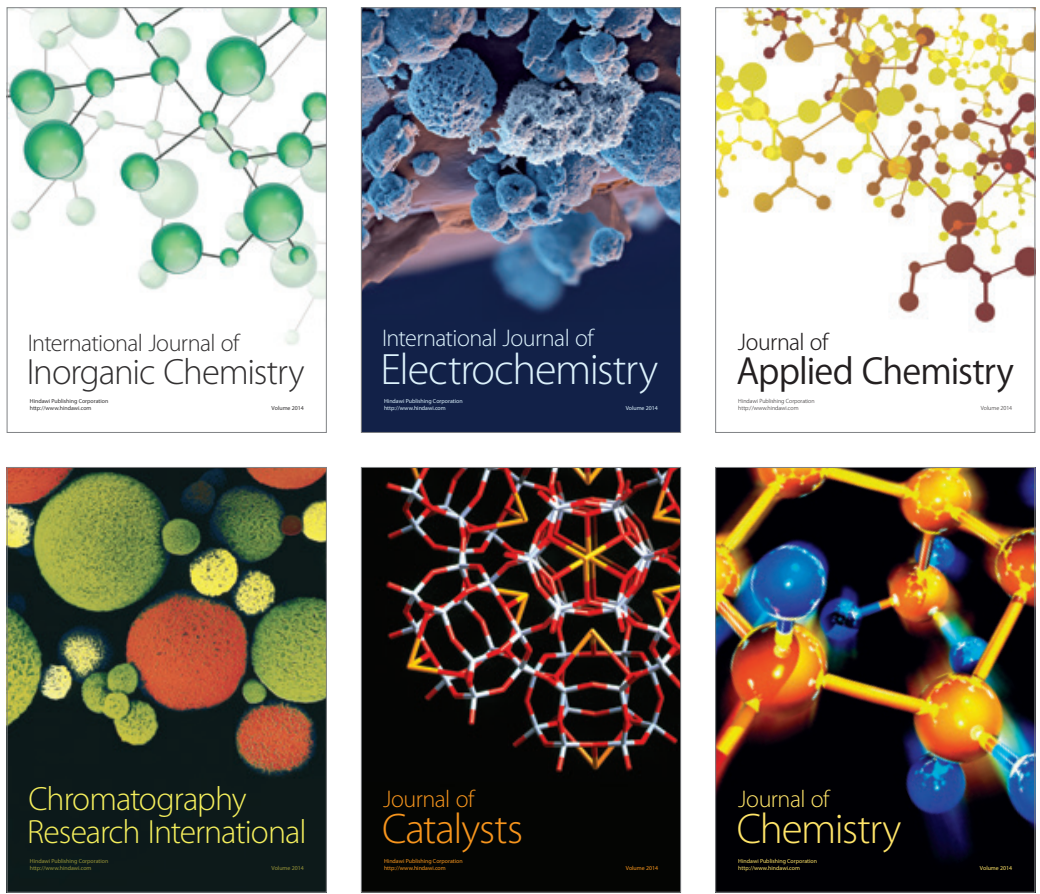

Journal of

Applied Chemistry
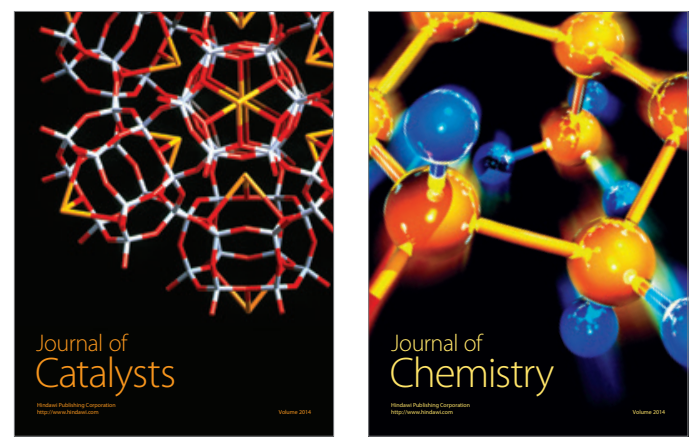
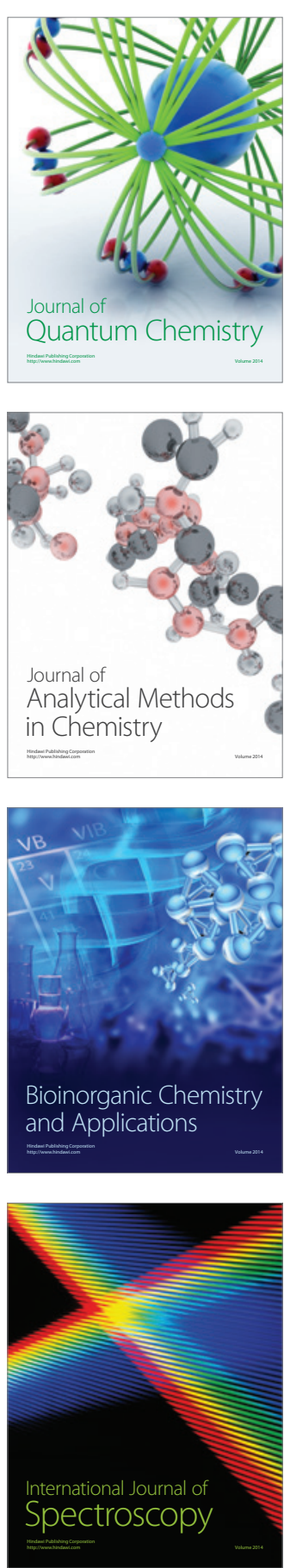